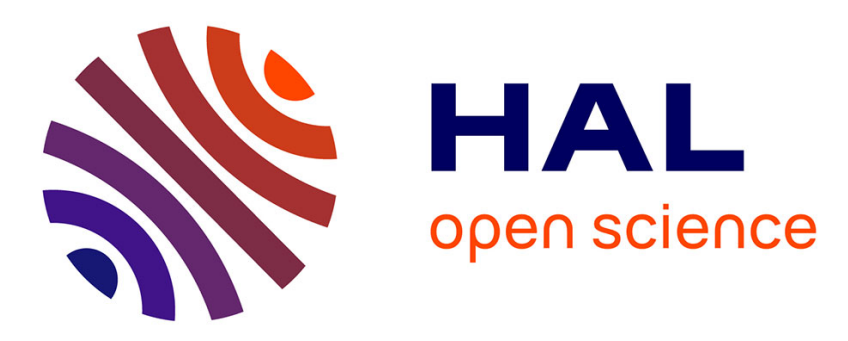

\title{
Influence of ion irradiation on switching field and switching field distribution in arrays of Co/Pd-based bit pattern media
}

Thomas Hauet, O Hellwig, S.-H Park, C Beigné, E Dobisz, B.D. Terris, D Ravelosona

\section{To cite this version:}

Thomas Hauet, O Hellwig, S.-H Park, C Beigné, E Dobisz, et al.. Influence of ion irradiation on switching field and switching field distribution in arrays of $\mathrm{Co} / \mathrm{Pd}$-based bit pattern media. Applied Physics Letters, 2011, 10.1063/1.3581896 . hal-01345352

\section{HAL Id: hal-01345352 https://hal.science/hal-01345352}

Submitted on 13 Jul 2016

HAL is a multi-disciplinary open access archive for the deposit and dissemination of scientific research documents, whether they are published or not. The documents may come from teaching and research institutions in France or abroad, or from public or private research centers.
L'archive ouverte pluridisciplinaire HAL, est destinée au dépôt et à la diffusion de documents scientifiques de niveau recherche, publiés ou non, émanant des établissements d'enseignement et de recherche français ou étrangers, des laboratoires publics ou privés. 


\title{
Influence of ion irradiation on switching field and switching field distribution in arrays of Co/Pd-based bit pattern media
}

\author{
T. Hauet, ${ }^{1,2, a)}$ O. Hellwig, ${ }^{1}$ S.-H. Park, ${ }^{3}$ C. Beigné, ${ }^{4}$ E. Dobisz, ${ }^{1}$ B. D. Terris, ${ }^{1}$ and \\ D. Ravelosona ${ }^{3}$ \\ ${ }^{1}$ Hitachi Global Storage Technologies, SJRC, San Jose, California 95135, USA \\ ${ }^{2}$ Institut Jean Lamour, UMR CNRS 7198, Nancy-Université, 54506 Vandoeuvre lès Nancy, France \\ ${ }^{3}$ Institut d'Electronique Fondamentale, UMR CNRS 8622, Université Paris-Sud, 91405 Orsay, France \\ ${ }^{4}$ DRFMC/SP2M, CEA Grenoble, 38054 Grenoble, France
}

(Received 19 February 2011; accepted 28 March 2011; published online 29 April 2011)

\begin{abstract}
We have used ion irradiation to tune switching field and switching field distribution (SFD) in polycrystalline $\mathrm{Co} / \mathrm{Pd}$ multilayer-based bit pattern media. Light $\mathrm{He}^{+}$ion irradiation strongly decreases perpendicular magnetic anisotropy amplitude due to $\mathrm{Co} / \mathrm{Pd}$ interface intermixing, while the granular structure, i.e., the crystalline anisotropy, remains unchanged. In dot arrays, the anisotropy reduction leads to a decrease in coercivity $\left(\mathrm{H}_{\mathrm{C}}\right)$ but also to a strong broadening of the normalized $\mathrm{SFD} / \mathrm{H}_{\mathrm{C}}$ (in percentage), since the relative impact of misaligned grains is enhanced. Our experiment thus confirms the major role of misorientated grains in SFD of nanodevice arrays. (C) 2011 American Institute of Physics. [doi:10.1063/1.3581896]
\end{abstract}

Today a major research effort in magnetism is targeted toward achieving ultrahigh density data storage with nanoscale magnets. Spin-transfer magnetic random access memory (spin-RAM) and bit patterned media (BPM) technologies are currently part of the most promising media. The implementation of both of these technologies relies on achieving in-detail physical understanding and control of the magnetization reversal mechanism in each nanoscopic individual bit to ensure reproducibility of the bit properties in order to avoid write errors.

Perpendicular magnetic anisotropy (PMA) materials, such as polycrystalline $\mathrm{Co} / \mathrm{Pd}, \mathrm{Co} / \mathrm{Pt}$, and $\mathrm{Co} / \mathrm{Ni}$ multilayers, are believed to be promising materials for both spin-RAM and BPM applications. ${ }^{1-4}$ Indeed, they have a well defined high amplitude uniaxial anisotropy that provides good thermal stability while offering low critical current in spintransfer devices ${ }^{2}$ and tunable switching fields in BPM. $^{4}$ However, full control of switching distribution [mentioned as switching field distribution (SFD) here, since our present experimental study involves a BPM system], remains unachieved. ${ }^{3,4}$ The SFD has multiple origins but intrinsic growth defects leading to anisotropy distribution has been shown to be a dominant one. ${ }^{5-8}$ These defects include misorientated grains, grain boundaries, distribution of easy axis angle, variations in lattice stress, and variations in thicknesses or interface roughness in multilayers. Two recent investigations ${ }^{7,8}$ indicate that misorientated crystalline grains are the major microstructural origin of SFD in (111) textured $[\mathrm{Co} / \mathrm{Pd}]$ multilayers. However, identifying the effects of these correlated structural defects remains an issue and key quest toward achieving lower SFD.

Here, we report on light $\mathrm{He}^{+}$ion irradiation as a tool to tune the switching field and SFD in textured (111) $[\mathrm{Co} / \mathrm{Pd}]_{8}$ full films and nanodot assemblies. Structural characterization confirms that ion irradiation does not affect the granular structure of the polycrystalline film, and therefore, does not affect the crystalline anisotropy. On the contrary, irradiation

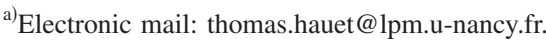

has a strong influence on the $\mathrm{Co} / \mathrm{Pd}$ interface anisotropy, inducing a reduction in the coercivity on both full films and BPM arrays. Besides, in the nanodot assemblies, the normalized SFD is increased dramatically, which can be understood from the enhanced relative impact of misorientated grains as the interlayer anisotropy is lowered with increasing ion dose.

$\mathrm{Ta}(15 \AA) / \operatorname{Pd}(30 \AA) /[\mathrm{Co}(2.8 \AA) / \operatorname{Pd}(9 \AA)]_{8} / \operatorname{Pd}(20 \AA)$ multilayers have been grown by magnetron sputtering at 3 mTorr Ar pressure onto both flat $\mathrm{Si}$ wafer and prepatterned substrates consisting of periodic arrays of $\mathrm{Si}$ pillars as described in detail earlier. ${ }^{3,5}$ These are polycrystalline systems with a highly (111) textured microstructure induced by the $\mathrm{Ta} / \mathrm{Pd}$ seed layers. ${ }^{9}$ The quality of the multilayering, in full films as well as in nanostructure arrays, has been shown in Ref. 3. A series of five identical $[\mathrm{Co} / \mathrm{Pd}]_{8}$ samples have been grown on both types of substrates (flat and prepatterned). One full film and one BPM sample are kept as-grown as reference samples and the others are irradiated at room temperature with $20 \mathrm{keV} \mathrm{He}{ }^{+}$ions at fluences of $5 \times 10^{14}$, 1 $\times 10^{15}, 2 \times 10^{15}$, and $3 \times 10^{15}$ ions $/ \mathrm{cm}^{2}$, respectively. ${ }^{10,11}$ At these energies, all ions penetrate through the multilayer and stop deep within the $\mathrm{Si}$ substrate. The full film structural properties have been analyzed by x-ray diffraction (XRD) and their magnetic properties have been measured using a Quantum Design vibrating sample magnetometer. The BPM magnetic features were explored using the polar magnetooptical Kerr effect (PMOKE) with a wavelength of $633 \mathrm{~nm}$ and a focal spot size of $20 \mu \mathrm{m}$. All experiments were performed at room temperature.

Comparison between grazing incidence $\mathrm{x}$-ray reflectivity (XRR) experiments performed on the full film samples [Fig. 1(a)] confirm the initial metallic stack thickness and do not show any clear evolution of the stack thickness or roughness as a function of the irradiation fluencies. The position of the superlattice peak, enlarged in Fig. 1(b), corresponds to a $[\mathrm{Co} / \mathrm{Pd}]$ period of $11.5 \AA$. The amplitude of the superlattice peak decreases with irradiation fluencies, which confirms the chemical interdiffusion between $\mathrm{Co}$ and $\mathrm{Pd}$ within the multilayer. Large angle XRD rocking curve and Bragg data 

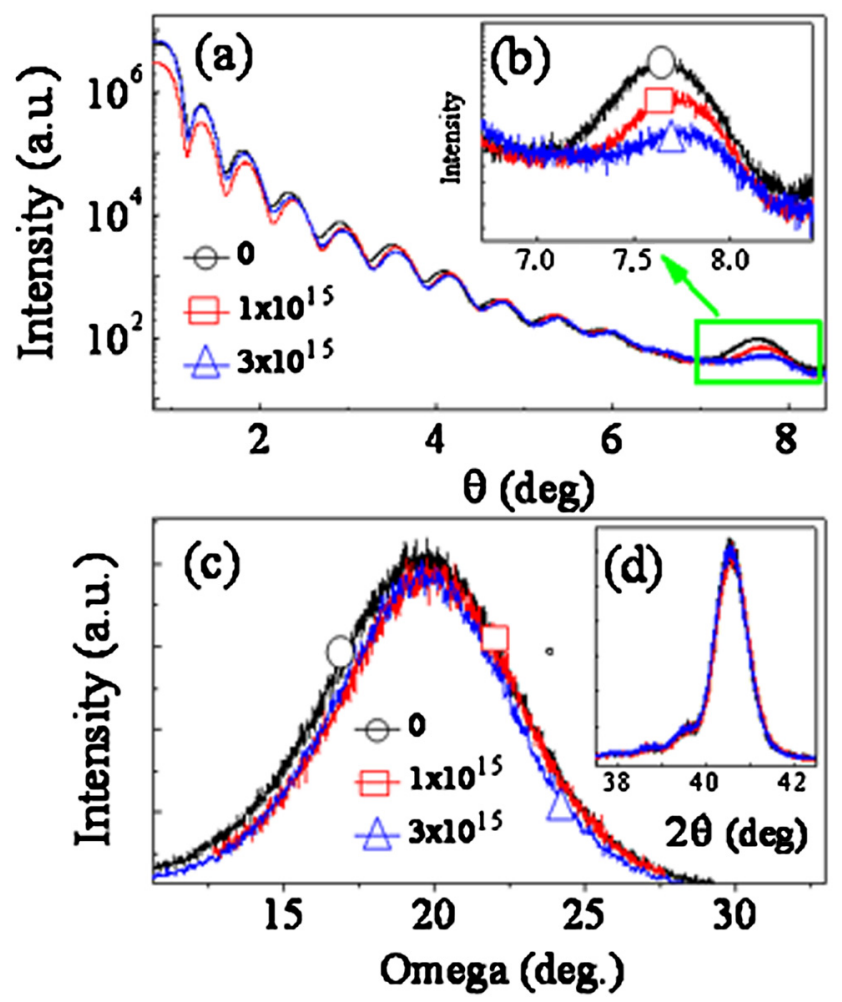

FIG. 1. (Color online) $[(\mathrm{a})-(\mathrm{d})] \mathrm{X}$-ray analysis of $[\mathrm{Co} / \mathrm{Pd}]_{8}$ irradiated multilayers having been exposed to 0 (black circles), $1 \times 10^{15}$ (red square) and $3 \times 10^{15}$ (blue triangle) ions $/ \mathrm{cm}^{2}$. (a) and (b) are grazing incidence XRR curves, while (c) shows rocking curves of the Co/Pd (111) Bragg peak displayed in the inset (d).

reveal the out-of-plane (111) texture of [Co/Pd] [Fig. 1(c)] with an average lattice parameter $\mathrm{d}_{(111)}$ of $2.22 \AA$ that is not affected by the irradiation [Fig. 1(d)]. The rocking curves of the Co/Pd (111) peak in Fig. 1(c) show a mosaic spread with a full width at half maximum (FWHM) that slightly diminishes from $7.2^{\circ}$ to $6.6^{\circ}$. with irradiation. The structural results obtained on the reference multilayer are in good agreement with previous studies ${ }^{3}$ and our findings on irradiated samples are coherent with past studies demonstrating that the major structural effect of irradiation with light $\mathrm{He}^{+}$ions is intermixing at interfaces without creation of crystalline orientation disorder. ${ }^{10-13}$ Particularly, it has been clearly established that the initial crystallographic structure and microstructure (grain size) is maintained, no surface roughness resulting from sputtering and no ion implant into the magnetic layer are induced.

Influence of ion irradiation on the $[\mathrm{Co} / \mathrm{Pd}]$ full film magnetic properties is quantified by measuring coercivity from perpendicular easy axis hysteresis loops and anisotropy from in-plane hard axis loops as a function of the ion fluence. The full film hysteresis loops are nicely square up to $2 \times 10^{15}$ ions $/ \mathrm{cm}^{2}$ [Fig. 2(a)] with sharp switching, indicating an easy domain wall propagation during the reversal, i.e., a low density of magnetic pinning sites. As shown in Fig. 2(a) and inset of Fig. 2(b), the full film coercivity decreases when increasing the ion dose. The same behavior is found for the anisotropy measurements [Fig. 2(b)]. At a fluence of $3 \times 10^{15}$ ions $/ \mathrm{cm}^{2}$, the sign of the anisotropy constant becomes negative, indicating a rotation from PMA to in-plane anisotropy. Note that the magnetization at saturation (around
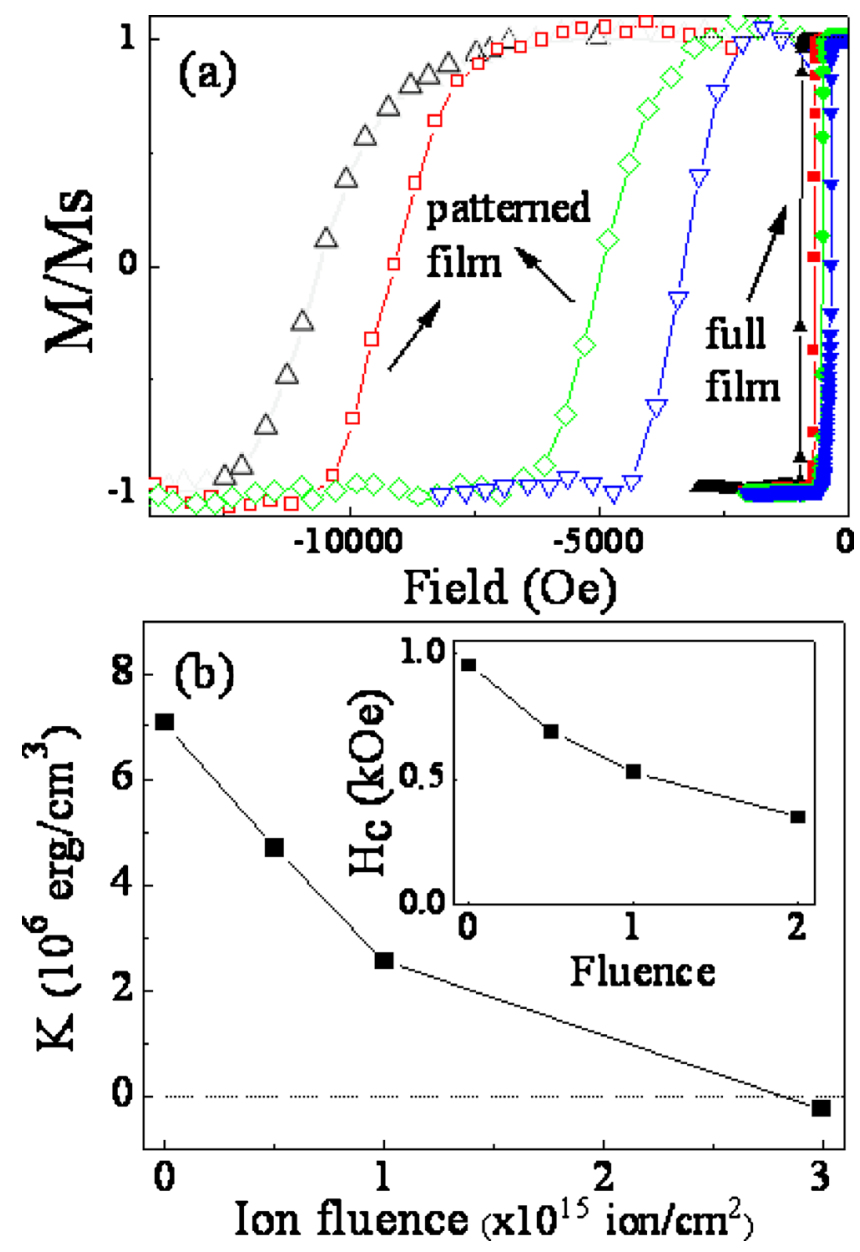

FIG. 2. (Color online) (a) Normalized magnetization vs field loops for $[\mathrm{Co} / \mathrm{Pd}]$ irradiated multilayers having been exposed to 0 (black triangles), $5 \times 10^{14}$ (red squares), $10^{15}$ (green diamonds), and $2 \times 10^{15}$ ions $/ \mathrm{cm}^{2}$ (blue reversed triangles) in the case of continuous films (solid symbols) and patterned nanodots (open symbols). (b) Anisotropy amplitude and coercivity (inset) of $[\mathrm{Co} / \mathrm{Pd}]_{8}$ continuous film as a function of the ion irradiation dose.

$500 \mathrm{emu} / \mathrm{cc})$ stays more or less constant $( \pm 50 \mathrm{emu} / \mathrm{cc})$ over the whole dose range.

Figure 3(a) summarizes results of the switching field and SFD for BPM samples at $100 \mathrm{~Gb} / \mathrm{in}^{2}$ (80 nm period) and $300 \mathrm{~Gb} /$ in. $^{2}$ (45 $\mathrm{nm}$ period). The switching field corresponds to the coercivity field of the PMOKE reversal curve as shown in Fig. 2(a). The normalized SFD is calculated as the FWHM of a Gaussian fit to the reversal curve derivative, divided by the coercivity. Only irradiation fluences that maintain positive anisotropy constant are considered (i.e., up to $2 \times 10^{15} \mathrm{ion} / \mathrm{cm}^{2}$ ). As expected from the irradiation induced PMA reduction, $\mathrm{H}_{\mathrm{C}}$ continuously decreases as the ion fluence increases, for both BPM densities. In parallel, the SFD is also decreasing but with a much shallower slope [Fig. 3(b)]. Therefore, the normalized SFD (i.e., SFD/ $\mathrm{H}_{\mathrm{C}}$ in percentage) is continuously growing as the dose increases [Fig. 3(c)].

To determine the origin of the influence of irradiation on SFD, one has to investigate the dot-to-dot anisotropy variations. In the Co/Pd grains, the anisotropy field is a combination of interface anisotropy (first order term), crystalline anisotropy (second order term) and demagnetization field. The interface anisotropy and demagnetization terms do not vary with the crystalline orientation of the grains. ${ }^{14}$ However, the crystalline anisotropy is uniaxial PMA for (111) texture, cu- 


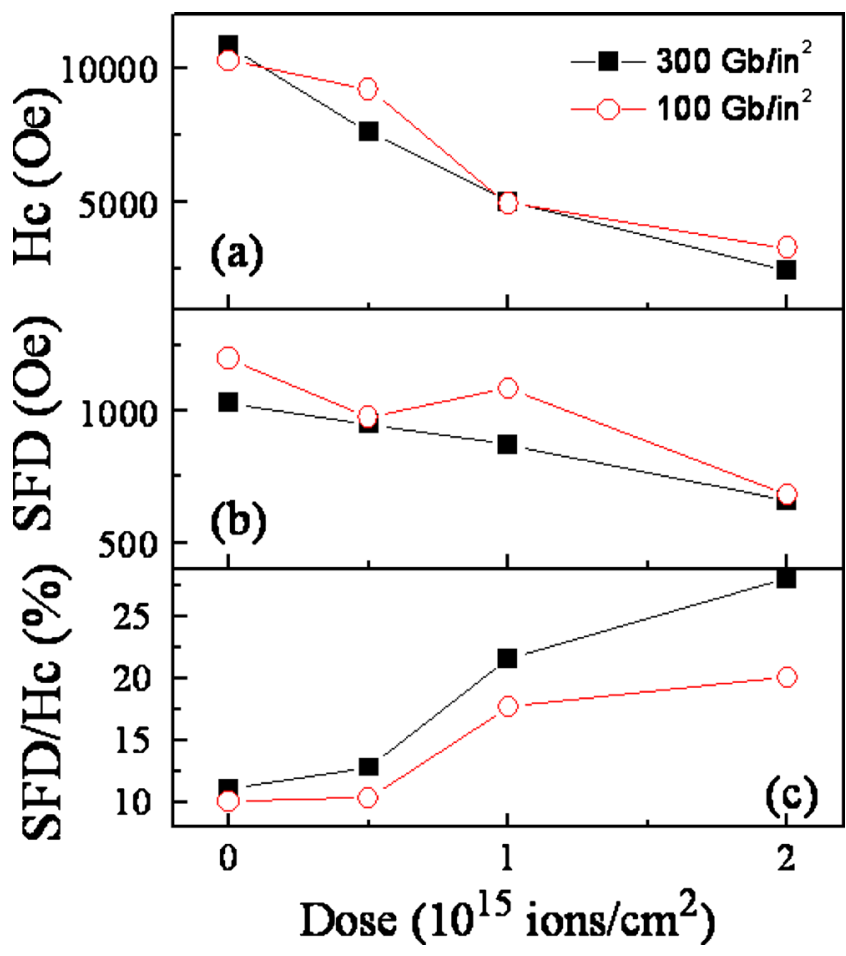

FIG. 3. (Color online) Coercivity (a), SFD (b), and normalized SFD (c) as a function of ion irradiation fluence for BPM samples with densities of $100 \mathrm{~Gb} /$ in. $^{2}$ (open red circles) and $300 \mathrm{~Gb} /$ in $^{2}$ (black squares).

bic for (110) and in-plane anisotropy for (100). Therefore, as demonstrated by Engel et al. ${ }^{14}[\mathrm{Co}(2.8 \AA) / \operatorname{Pd}(9 \AA)]$ total anisotropy evolves from about $1 \times 10^{7} \mathrm{erg} / \mathrm{cm}^{3}$ in the case of (111) crystalline orientation to almost zero in the case of a (100) orientation. As a consequence, the larger the number of (100) and (110) grains per dot is, the lower is the switching field. ${ }^{7,8}$ Note that the reversal fields of the BPM dots are systematically lower than the effective anisotropy, even before irradiation [Fig. 3(a)]. The reference sample has $\mathrm{H}_{\mathrm{K} \text {,eff }}$ $=2 \mathrm{~K} / \mathrm{M}_{\mathrm{s}}-4 \pi \mathrm{M}_{\mathrm{s}}=22 \mathrm{kOe}$, where $\mathrm{M}_{\mathrm{s}}$ is the magnetization at saturation and $\mathrm{K}$ is the anisotropy constant shown in Fig. 2(b). This discrepancy with the Stoner-Wohlfarth theory mostly originates from non uniform reversal modes that are initiated in low anisotropy regions like misorientated grains (and thermal activation). ${ }^{5}$ Besides, as far as SFD is concerned, chemical interfaces represent the leading anisotropy term, however, the layering has very little irregularities or defects, so it is only a second order contribution to the SFD. In contrast the crystalline orientation of the grains is a second order anisotropy term, while misaligned grains and corresponding variations in anisotropy generate the dominating term to the SFD.

From the $\mathrm{Co} / \mathrm{Pd}$ initial features, ion irradiation significantly decreases the interface anisotropy due to chemical intermixing, while the crystalline anisotropy and demagnetization fields are not affected. As stated above, no additional defects are induced in the magnetic layer by the irradiation. As the chemical layering is destroyed, the leading term in the anisotropy is lost and $\mathrm{H}_{\mathrm{C}}$ decreases dramatically with ion dose. At the same time the SFD (in Oe) is only decreased very little since the layering was very regular to start with and also the intermixing due to ion irradiation is quite uniform for all areas. As a result of such a strong decrease in $\mathrm{H}_{\mathrm{C}}$, but only a very modest decrease in SFD, we obtain a strong increase in relative $\mathrm{SFD} / \mathrm{H}_{\mathrm{C}}(\%)$. This explanation is consistent with the fact that we observe a larger impact of irradiation for the $300 \mathrm{~Gb} /$ in. $^{2}$ array as compared to the $100 \mathrm{~Gb} /$ in. $^{2}$ one [Fig. 3(c)], since the impact of low anisotropy defects is statistically stronger as the dot size decreases. $^{5}$

In conclusion, we have performed moderate $\mathrm{He}^{+}$ion irradiation experiments on textured (111) $[\mathrm{Co} / \mathrm{Pd}]_{8}$ systems for full films and nanodot assemblies up to $300 \mathrm{~Gb} / \mathrm{in}^{2}{ }^{2}$. Ion irradiation strongly decreases perpendicular anisotropy (PMA) due to interface intermixing between Co and Pd layers, while the granular and crystalline structure remains unchanged. According to the PMA reduction, a large decrease in BPM coercivity is found, with only a very modest decrease in the absolute SFD value. Therefore, the normalized SFD increases quite dramatically with ion dose. Given that ion irradiation only destroys the chemical interfaces, we can understand these results from the fact that the chemical interlayering provides the leading contribution to $\mathrm{H}_{\mathrm{C}}$, but only the second order term to the SFD, while the crystalline structure is the leading contribution to the SFD (misaligned grains) but second order to $\mathrm{H}_{\mathrm{C}}$ and anisotropy. Overall our results confirm that ion irradiation can be used to systematically tailor the magnetic properties of PMA multilayer-based nanostructures, although in our case it leads to an increase in normalized SFD. Finally, our experiments demonstrate the major role of misorientated grains in SFD of PMA multilayer-based BPM, as recently proposed in Refs. 7 and 8.

${ }^{1}$ S. Mangin, D. Ravelesona, J. A. Katine, M. J. Carrey, B. D. Terris, and E. E. Fullerton, Nature Mater. 5, 210 (2006).

${ }^{2}$ S. Mangin Y. Henry, D. Ravelosona, J. A. Katine, and E. E. Fullerton, Appl. Phys. Lett. 94, 012502 (2009).

${ }^{3}$ O. Hellwig, T. Hauet, T. Thomson, E. Dobisz, J. D. Risner-Jamtgaard, D. Yaney, B. D. Terris, and E. E. Fullerton, Appl. Phys. Lett. 95, 232505 (2009).

${ }^{4}$ T. Hauet, E. Dobisz, S. Florez, J. Park, B. Lengsfield, B. D. Terris, and O. Hellwig, Appl. Phys. Lett. 95, 262504 (2009).

${ }^{5}$ T. Thomson, G. Hu, and B. D. Terris, Phys. Rev. Lett. 96, 257204 (2006).

${ }^{6}$ O. Ozatay, T. Hauet, S. H. Florez, J. A. Katine, A. Moser, J. U. Thiele, L. Folks, and B. D. Terris, Appl. Phys. Lett. 95, 172502 (2009).

${ }^{7}$ J. W. Lau, R. D. McMichael, S. H. Chung, J. O. Rantschler, V. Parekh, and D. Litvinov, Appl. Phys. Lett. 92, 012506 (2008).

${ }^{8}$ J. M. Shaw, H. T. Nembach, T. J. Silva, S. E. Russek, R. Geiss, C. Jones, N. Clark, T. Leo, and D. J. Smith, Phys. Rev. B 80, 184419 (2009).

${ }^{9}$ J. M. Shaw, W. H. Rippard, S. E. Russek, T. Reith, and C. M. Falco, J. Appl. Phys. 101, 023909 (2007).

${ }^{10}$ C. Chappert, H. Bernas, J. Ferré, V. Kottler, J.-P. Jamet, Y. Chen, E. Cambril, T. Devolder, F. Rousseaux, V. Mathet, and H. Launois, Science 280, 1919 (1998).

${ }^{11}$ T. Devolder, Phys. Rev. B 62, 5794 (2000).

${ }^{12}$ V. Parekh, D. Smith, E. Chunsheng, J. Rantschler, S. Khizroev, and D. Litvinov, J. Appl. Phys. 101, 083904 (2007).

${ }^{13}$ J. Fassbender, D. Ravelosona, and Y. Samson, J. Phys. D: Appl. Phys. 37, R179 (2004).

${ }^{14}$ B. N. Engel, C. D. England, R. A. Van Leeuwen, M. H. Wiedmann, and C. M. Falco, Phys. Rev. Lett. 67, 1910 (1991). 